\title{
Acción protectora del ácido kaurénico en el estrés oxidativo hepático
}

\author{
Mendoza, C. ; Márquez, A. ${ }^{2}$; Matheus, N.; Sosa, S.M.; López-Ortega, A. ${ }^{1}$ \\ 'Univ. Centroccidental "Lisandro Alvarado”, Depto. Ciencias Básicas, Decanato de Ciencias Veterinarias, \\ Unidad de Investig. Cs. Funcionales "Dr. Haity Moussatché”, Área Biol. Molec.y Celular; \\ ${ }^{2}$ Área Fisiología Animal, Barquisimeto, Venezuela. \\ E-mail: carmenmendoza@ucla.edu.ve
}

\begin{abstract}
Resumen
Mendoza, C.; Márquez, A.; Matheus, N.; Sosa, S.M.; López-Ortega, A.: Acción protectora del ácido kaurénico en el estrés oxidativo hepático. Rev. vet. 28: 1, 27-30, 2017. El ácido kaurénico es un antioxidante extraído del "frailejón", la planta más representativa de la flora de los páramos andinos de Venezuela, Colombia y norte de Ecuador. Se ha reportado que tal diterpenoide posee efecto protector frente al estrés oxidativo. El objetivo de este estudio fue determinar la acción antioxidante del ácido kaurénico y establecer su efecto protector ante la generación del hígado graso experimental causado por el tóxico etionina. Se utilizaron 24 ratones hembras NMRI. Al grupo experimental se le administró vía i.p. $20 \mathrm{mg} / \mathrm{kg}$ de ácido kaurénico durante 15 días El grupo control sólo recibió vehículo. A los 13 días post-inoculación se les indujo hígado graso a ambos grupos mediante etionina i.p. $(7,5 \mathrm{mg} / 20 \mathrm{~g})$, manteniéndose los animales en ayunas durante 48 horas. En el homogenizado de hígado se determinaron los niveles de triglicéridos, proteínas, malondialdehido y dienos conjugados, así como las actividades de superóxido dismutasa y glutatión peroxidasa. Los resultados fueron analizados mediante la prueba " $\mathrm{t}$ " de Student $(\mathrm{p}<0,05)$. Los datos sugieren que el ácido kaurénico actúa como antioxidante y disminuye la génesis de lipoperoxidación.
\end{abstract}

Palabras clave: ratón, estrés oxidativo, hígado graso, ácido kaurénico, antioxidante.

\begin{abstract}
Mendoza, C.; Márquez, A.; Matheus, N.; Sosa, S.M.; López-Ortega, A.: Protective action of kaurenic acid on oxidative stress in the liver. Rev. vet. 28: 1, 27-30, 2017. The "frailejón" is the main representative plant of the flora of the Andean Highlands of Venezuela, Colombia and Ecuador. It has been reported that kaurenic acid, a diterpenoid present in the plant, has protective effect against oxidative stress. The aim of this study was to determine the antioxidant effect of kaurenic acid and to establish its protective effect against the generation of experimental fatty liver caused by ethionine. Twenty four female NMRI mice were used. The experimental group was administered i.p. $20 \mathrm{mg} / \mathrm{kg}$ of kaurenic acid for 15 days while control group only received vehicle. Thirteen days after kaurenic acid administration, fatty liver was induced in both groups by administration of ethionine i.p. $(7.5 \mathrm{mg} / 20 \mathrm{~g})$ and fasting for 48 hours. Triglycerides, proteins, malondialdehyde and conjugated dienes were determined in liver homogenate, as well as the activity of superoxide dismutase and glutathione peroxidase. Results were analyzed using the " $\mathrm{t}$ " Student $(\mathrm{p}<0.05)$ test. Gathered data suggest that kaurenic acid acts as an antioxidant and reduces the genesis of lipid peroxidation.
\end{abstract}

Key words: mouse, oxidative stress, hepatic lipidosis, kaurenic acid, antioxidant.

\section{INTRODUCCIÓN}

El daño hepático producido durante la hepatoesteatosis representa un problema en las explotaciones ganaderas de Venezuela y otros países. Las consecuencias del hígado graso van desde una disminución de la producción lechera, hasta la alteración de los parámetros

Recibido: 20 mayo 2016 / Aceptado: 30 agosto 2016 productivos e incluso la pérdida por muerte súbita de bovinos de alto valor genético.

Las vitaminas antioxidantes $\mathrm{E}$ y $\mathrm{C}$ han resultado beneficiosas por su efecto protector al hígado graso, sin embargo su elevado costo limita su empleo. Es por esto que la utilización de un producto antioxidante alternativo como el ácido kaurénico, extraído del "frailejón", resultaría adecuado. 
El "frailejón" es la planta más representativa de la flora de los páramos andinos de Venezuela, Colombia y norte del Ecuador ${ }^{8}$. Las especies de esta planta están constituidas por el género Espeletia, creado por Humbolt y Bonpland ${ }^{7}$ y redefinido, muchos años más tarde, como subtribu Espeletiinae en función de la gran cantidad de especies encontradas ${ }^{1}$. Estas hierbas se caracterizan por su alto contenido en resinas y aceites esenciales, presentando un crecimiento espiralado en forma de roseta ${ }^{10}$; su resina contiene mezclas de diterpenos, los cuales protegerían a la planta contra las bajas temperaturas ${ }^{13}$.

Se han caracterizado diversos tipos de diterpenos, entre los cuales se encuentra el ácido kaurénico (entkaur-16-en-19-oico), de fórmula molecular $\mathrm{C} 20 \mathrm{H} 30 \mathrm{O} 2$ (1). Estos diterpenos fueron aislados de diferentes vegetales y considerados como compuestos biológicamente activos ${ }^{9}$. Investigaciones realizadas con este tipo de compuestos muestran un efecto citotóxico, contra las células A-549 del pulmón de seres humanos ${ }^{11}$. Se ha reportado su acción contra los cánceres de ovario y pulmón, así como su utilidad frente al melanoma maligno humano ${ }^{5,11}$. El ácido kaurénico ha demostrado poseer propiedades anti-inflamatorias y anti-anticonvulsivantes, entre otros efectos ${ }^{14}$.

Varias investigaciones detallan la acción del ácido kaurénico sobre la inhibición de la peroxidación lipídica y de la superóxido dismutasa. Los diterpenoides son efectivos agonistas del sistema biológico protector del estrés oxidativo ${ }^{6} \mathrm{y}$ tienen efecto cardioprotectivo disminuyendo la peroxidación lipídica inducida por radicales libres de oxígeno ${ }^{13}$.

El objetivo de esta investigación fue determinar el efecto del ácido kaurénico sobre la lipoperoxidación del hígado graso experimental en ratones hembras adultas.

\section{MATERIAL Y MÉTODOS}

Se emplearon 24 ratones hembras NMRI, provenientes del Bioterio Central de la UCLA, con peso promedio de $25 \mathrm{~g}$, mantenidos bajo condiciones estándares. Al grupo experimental, conformado por 12 animales, se le administró ácido kaurénico vía i.p. a razón de 20 $\mathrm{mg} / \mathrm{kg}$ durante 15 días y al grupo control -con igual número de animales- sólo vehículo.

El ácido kaurénico utilizado en el presente trabajo fue aislado de Espeletia semiglobulata ${ }^{12}$ y proporcionado en forma de kaurenato sódico $\left[\mathrm{C}_{20} \mathrm{H}_{29} \mathrm{NaO}_{2}\right]$, el cual fue disuelto en agua destilada deionizada antes de su uso.

A los 13 días de tratamiento se les indujo hígado graso a ambos grupos de roedores, mediante administración de etionina i.p. (7,5 mg/20 g). Tras mantenerse en ayunas durante 48 horas, todos los animales fueron sacrificados bajo ligera eterización, tras la cual se les disecó el hígado a 6 roedores experimentales y a 6 controles, para su homogenización en buffer tris-sacarosa $250 \mathrm{mM} \mathrm{pH} \mathrm{7,2.}$
En el sobrenadante se determinaron: a) triglicéridos (TG, mediante kit Qualitest con un patrón de 200 $\mathrm{mg} / \mathrm{dl}$ de trioleína); b) proteínas (P, por kit Bio Rad Lab, CA, USA) que utiliza como patrón albúmina sérica bovina $1,41 \mathrm{mg} / \mathrm{ml}$; c) malondialdehido (MDA, por la técnica de TBARS, Ohkawa 1978); y dienos conjugados (DC, Wallin 1993).

El resto de los animales ( 6 controles y 6 experimentales) fue sacrificado bajo ligera eterización, el hígado se canuló a través de la vena porta para perfundir solución fisiológica fría con EDTA al 0,1\%. El órgano se disecó y pesó, luego un trozo de hígado se homogenizó con sacarosa $0,25 \mathrm{M}$ y se diluyó al $10 \% \mathrm{p} / \mathrm{v}$ para ser centrifugado a $12.000 \mathrm{rpm}\left(4^{\circ} \mathrm{C}\right)$ durante 10 minutos.

El sobrenadante se separó con pipeta Pasteur y se determinó la actividad superóxido dismutasa (SOD) mediante kit de Lab. Calbiochem. Otro trozo de tejido fue homogenizado con una solución buffer provisto por el kit Calbiochem y en el sobrenadante se determinó la actividad de la glutatión peroxidasa (GP).

Los resultados obtenidos fueron analizados por el paquete estadístico SPSS, versión 17.0 para Windows. Se realizó la estadística descriptiva de las variables y los resultados se expresaron como media aritmética \pm error estándar (ES). La comparación de los valores medios entre los grupos experimental y control se efectuó a través de una prueba " $t$ " de Student $(p<0,05)$.



Figura 1. Concentración hepática de triglicéridos (mg de TG/mg de proteínas) en ambos lotes ( $\overline{\mathrm{X}} \pm \mathrm{ES}$, $* p<0,05)$.



Figura 2. Concentración hepática de triglicéridos (mg de TG/g de tejido húmedo) en ambos lotes ( $\overline{\mathrm{X}} \pm \mathrm{ES}$, $* * \mathrm{p}<0,025)$. 


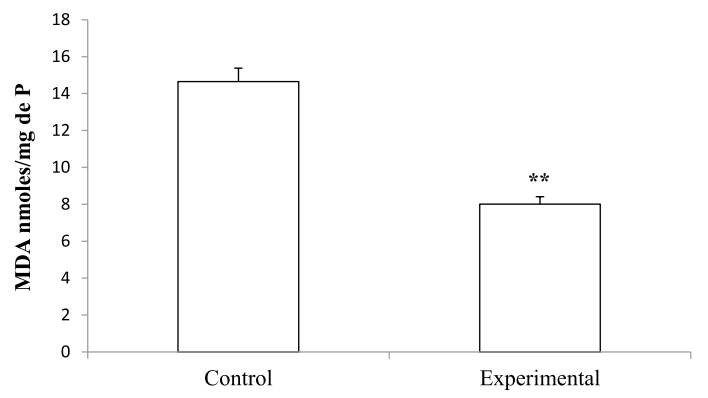

Figura 3. Concentración hepática de malondialdehido (MDA, nmol/mg de proteínas), $\overline{\mathrm{x}} \pm \mathrm{ES}\left({ }^{* *} \mathrm{p}<0,025\right)$.

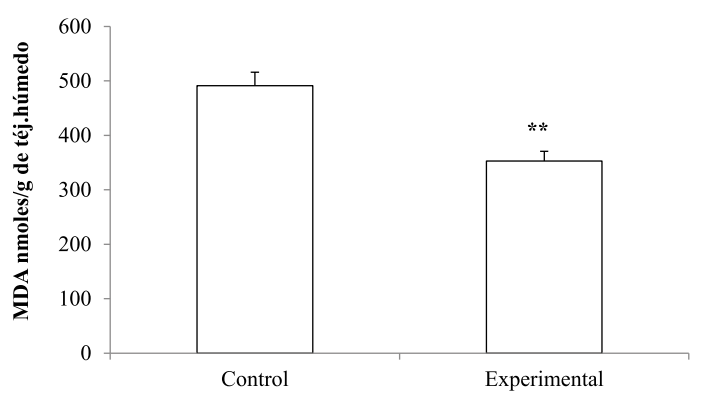

Figura 4. Concentración hepática de malondialdehido (MDA, nmol/g de tejido húmedo) en ambos lotes $\left(\overline{\mathrm{x}} \pm \mathrm{ES},{ }^{* *} \mathrm{p}<0,025\right)$.

\section{RESULTADOS Y DISCUSIÓN}

En los animales tratados con ácido kaurénico se obtuvo una disminución muy significativa de los niveles de triglicéridos, medidos en homogeneizados del hígado, al ser comparados con los roedores del grupo control (Figuras 1 y 2).

En coincidencia, otros autores afirman que en situaciones de estrés oxidativo se manifiestan lesiones producidas por radicales libres, que reaccionan químicamente con lípidos, proteínas, carbohidratos y ADN en el interior de las células, así como contra componentes de la matriz extracelular, por lo que pueden desencadenar un daño irreversible que, si es muy extenso, puede conducir a la muerte celular ${ }^{2}$.

En cuanto al grado de estrés oxidativo, se pudo apreciar un efecto hepatoprotector del ácido kaurénico, evidenciado por una disminución de los niveles de MDA en el grupo experimental, al ser comparado con el grupo control. Esta diferencia se mantuvo al expresar los resultados en nmoles de MDA/gramo de tejido húmedo (Figuras 3 y 4).

En el caso de los dienos conjugados, producto inicial del proceso de lipoperoxidación, se observó una disminución de los mismos en los animales experimentales con respecto a los controles, tanto cuando se expresaron por mg de proteínas, como por gramo de tejido húmedo (Figuras 5 y 6 ).

Con respecto a las enzimas antioxidantes, los resultados obtenidos indicaron que la actividad de SOD hepática, expresada en U/ml (actividad catalítica) o en
U/mg PT (actividad especifica), estuvo significativamente aumentada $(\mathrm{p}=0,05)$ en el grupo inyectado con ácido kaurénico (Figuras 7 y 8). En forma similar, se obtuvo un aumento significativo en la actividad de la GP (Figura 9).

En base a los resultados obtenidos es posible indicar que todos los parámetros medidos fueron signi-



Figura 5. Concentración hepática de dienos conjugados (DC, moles/mg de proteína), $\overline{\mathrm{x}} \pm \mathrm{ES}\left({ }^{*} \mathrm{p}<0,025\right)$.

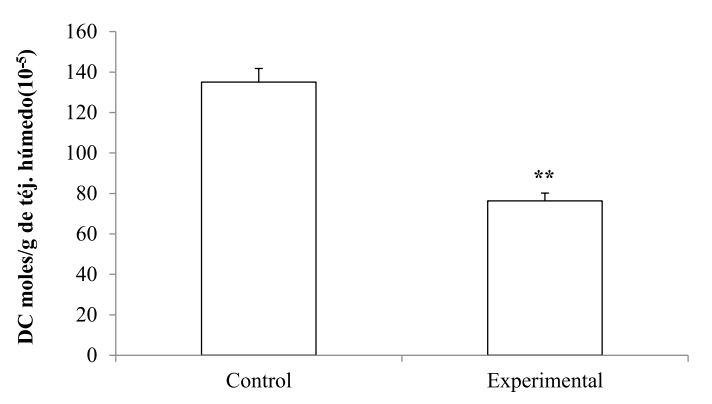

Figura 6. Concentración hepática de dienos conjugados (DC, moles/g tejido húmedo), $\bar{x} \pm \mathrm{ES}\left({ }^{*} \mathrm{p}<0,025\right)$.

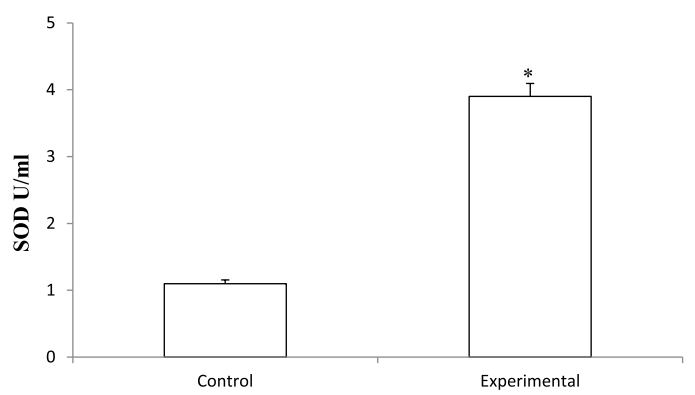

Figura 7. Concentración hepática de superóxido dismutasa (SOD, U/ml), $\bar{x} \pm \mathrm{ES},\left({ }^{*} \mathrm{p}<0,05\right)$.



Figura 8. Concentración hepática de superóxido dismutasa (SOD, $\mathrm{U} / \mathrm{ml}$ de proteína), $\overline{\mathrm{x}} \pm \mathrm{ES}\left({ }^{*} \mathrm{p}<0,05\right)$. 


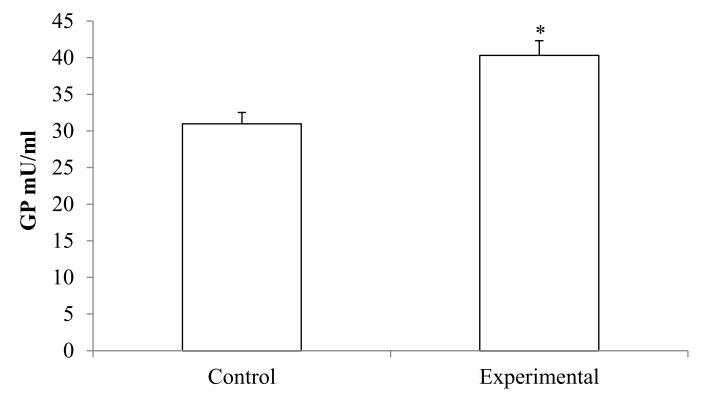

Figura 9. Concentración hepática de glutation peroxidasa (GP, $\mathrm{mU} / \mathrm{ml}), \overline{\mathrm{x}} \pm \mathrm{ES}\left({ }^{*} \mathrm{p}<0,05\right)$.

ficativamente más bajos en los animales a quienes se les administró ácido kaurénico, en comparación con el grupo control. La acción antioxidante de los ent-kaurenos ha sido estudiada previamente; así, rabdosin-B y oridonin han sido reportados como potentes inhibidores de la peroxidación lipídica ${ }^{4}$.

Los presentes hallazgos concuerdan con lo reportado por investigaciones que indican que el diterpenoide extraído de Podocarpus nagi es un efectivo agonista del sistema biológico protector del estrés oxidativo celular ${ }^{6}$.

También se ha demostrado que el ácido kaurénico posee acción antinflamatoria, evidenciada por una disminución en la producción de MDA al inhibir las actividades de cicloxigenasa y tromboxanos ${ }^{3}$. En este trabajo también se comprobó el efecto cardioprotector del kaureno, determinado por una reducción de la peroxidación lipídica mediada por los radicales libres.

En conclusión, surge que el ácido kaurénico ejerce un efecto protector ante el estrés oxidativo del hígado graso, evidenciado por una disminución de la concentración de MDA y de los DC en el tejido hepático, hallazgo relacionado con una mayor actividad de las enzimas SOD y GP. Así, se establece la acción antioxidante de este diterpenoide extraído del "frailejón", comprobándose que el menor grado de estrés oxidativo hepático trajo como consecuencia una disminución de la cuantía de la hepatoesteatosis, evidenciada por una menor concentración de los triglicéridos hepáticos.

Agradecimientos. Este trabajo fue financiado por el Consejo de Desarrollo Científico, Humanístico y Tecnológico (CDCHT) de la Universidad Centroccidental Lisandro Alvarado (Venezuela). Los autores agradecen al Dr. Alfredo Usubillaga, Universidad de Los Andes, por la donación de la droga ensayada y al Sr. Orlando Linarez por su asistencia técnica.

\section{REFERENCIAS}

1. Cuatrecasas J. 1976. A new subtribe in the Heliantheae (Compositae): Espeletiinae. Phytologia 35: 43-61.

2. Galván T, Guisado BR, García M, Ochoa J. 2008. Antioxidantes y ejercicio físico: funciones de la melatonina. Rev Andaluza Med Deport 1: 61-72.

3. Ghisalberti EL. 1997. The biological activity of naturally occurring kaurane diterpenes. Fitotherapy 97: 303-325.

4. Guoan L, Lan D, Yan Y, Hong Y, Qingming Y, Hanqing W. 2006. Anti-oxidative action of ent-kaurene diterpenoids. Res Chem Intermed 32: 787-794.

5. Haraguchi H, Saito T, Okamura N, Yagi A.1995. Inhibition of lipid peroxidation and superoxide generation by diterpenoids from Rosmarinus officinalis. Plant Med 61: 333-336.

6. Haraguchi S, Good RA, Cianciolo GJ, Engelman RW, Day NK. 1997. Immunosuppressive retroviral peptides: immunopathological implications for immunosuppressive influences of retroviral infections. J Leukoc Biol 61: 654666.

7. Humboldt \& Bonpland. 1808. Espeletia Mutis. Pl Aequinoct 2: 70-72.

8. Monasterio M. 1980. Las formaciones vegetales de los páramos de Venezuela. En: Estudios ecológicos en los páramos andinos (Monasterio M. Ed), Edit Univ de Los Andes, Mérida, Venezuela, p. 93-158.

9. Okuyama E, Nishimura S, Yamazaki M. 1991. Analgesic principles from Aralia cordala thunb. Chem Pharm Bull Tokyo 39: 405-407.

10. Standley PC. 1915. The genus Espeletia. Amer J Bot 2: 468-486.

11. Sun H, Lin Z, Niv F, Lin L, Chai H, Pezzuto J, Cordell G. 1995. Citotoxic ent- kaurene diterpenoids from three Isodon species. Phytochemistry 38: 437-442.

12. Usubillaga A, Capra MC. 1987. Chemical constituents of Espeletia semiglobulata. Fitoterapia LIX: 383-384.

13. Usubillaga A, Morales A. 1972. Kaurenic acids in Espeletia species. Phytochemistry 11: 1856-1857.

14. Zhang CF, Wang DS, Ling XZ. 1996. Diacetylamethystoidin; a protects isolated working rat heart against cardial reperfusion injury. Publ Dept Pharm, Jining Medical College, China, 17: 245-248. 